\title{
DISTRIBUTED FORMATION CONTROL WITH TIME AND CONNECTIVITY CONSTRAINTS
}

\author{
María Guinaldo, José Sánchez, Sebastián Dormido \\ UNED, Juan del Rosal 1628040 Madrid, \{mguinaldo,jsanchez,sdormido\}@dia.uned.es \\ Dimos V. Dimarogonas \\ KTH Royal Institute of Technology, SE-100 44, Stockholm, Sweden, dimos@kth.se
}

\begin{abstract}
In this paper, we propose a distributed control law for non-holonomic vehicles that guarantees to achieve the desired formation and location before a given deadline, while maintaining the connectivity of the group. The group is commanded by a a selected subset of the agents, which know the location of the desired objective, while the rest of the vehicles only have information about their relative desired positions respect their set of neighbors. The analytical results are illustrated with a simulation example.
\end{abstract}

Palabras clave: Multi-agent systems, formation control, distributed control, connectivity.

\section{INTRODUCTION}

Significant developments in the fields of communication technology, wireless technology, embedded devices, and many others, have enabled the development of autonomous air, ground, or underwater vehicles. Groups of such vehicles, referred to as agents, can be utilized to solve a variety of problems very efficiently, such as for example exploration and monitoring tasks $[3,9,11]$.

One example of a group objective for multi-agent systems is state agreement or consensus, i.e., all agents are supposed to converge to a common point or state. Such consensus problems have a variety of applications in flocking, attitude synchronization in satellite swarms, distributed sensor networks, congestion control in communication networks, or formation control [8]. We are particularly interested in the last field of application since achieving a stable formation is analogous to reaching consensus.

Another way of interaction in a multi-agent systems is when there are the so-called leader agents, whose dynamics need not conform to those of the non-leader (follower) agents. In these setups, a selected subset of the agents are following a tasklevel controller that encodes the transport of the network from one location to another. The rest of the agents are executing a simple local interaction- based control strategy for keeping the team together. The motivation of such configuration is that it relieves resources since only the leader agents are able to tell global positions and/or positions relative to particular landmarks, thus limiting the required sensor load of the remaining agents [4].

It is well known that a general connectivity analysis of an arbitrary network is extremely complex, and a lot of results have appeared recently regarding maintaining connectivity in networks of homogeneous agents $[10,12,14]$ and heterogeneous agents [15], to cite a few. In this paper, we address the problem of connectivity maintenance but, additionally, the group of agents has to perform a task before some given deadline. This time constraint imposes an additional requirement to the group since it has to travel together to maintain the connectivity but fast enough to reach the objective in time, considering that only the leader(s) know the final destination of the system. Whereas finite-time consensus algorithms [2, 13] can guarantee the achievement of a formation in finite-time and predict the deadline based on initial conditions and algebraic properties, basically, the idea here is different: The time constraint is given and it is the design of the control law that guarantees its satisfaction.

Moreover, in this paper, instead of modeling agents as single or double integrator such as in the papers cited above, we consider non-holonomic vehicles that move in the plane, which is a more realistic model for mobile robots. The team of robots, commanded by the leader(s) needs to reach a formation around a specific point in the state space, only known by the leader(s), while the rest of the the agents only have information about relative distances. Then, the leader agents have to command the team of robots to reach the objective before some given deadline.

The remainder of the paper is as follows. In Section 2 we state the required preliminaries. The problem is formulated in Section 3. In Section 4 we describe the proposed solution and the analytical results of the paper are provided. In Section 5 a simulation example illustrates the results. Fi- 
nally, a discussion about the results concludes the paper is Section 6 .

\section{PRELIMINARIES}

Consider a set of $N$ agents. The topology of the multi-agent system can be modeled as a static undirected graph $\mathcal{G}=(\mathcal{V}, \mathcal{E})$, where $\mathcal{V}$ is the set of nodes and $\mathcal{E}$ is the set of edges, which denotes the communication capability between the respective agents. For each agent $i, \mathcal{N}_{i}$ represents the neighborhood of $i$, i.e., $\mathcal{N}_{i}=\{j \in \mathcal{V}:(i, j) \in \mathcal{E}\}$. A subgraph $\mathcal{G}_{s}$ of an undirected graph $\mathcal{G}$ is an undirected graph such that the vertex set $\mathcal{V}\left(\mathcal{G}_{s}\right) \subseteq$ $\mathcal{V}(\mathcal{G})$ and the edge set $\mathcal{E}\left(\mathcal{G}_{s}\right) \subseteq \mathcal{E}(\mathcal{G})$. Let $\mathcal{G}_{1}$ and $\mathcal{G}_{2}$ be subgraphs of $\mathcal{G}$. We say that $\mathcal{G}_{1}$ and $\mathcal{G}_{2}$ are disjoint if they have no vertex in common, and edge-disjoint if they have no edge in common. The union $\mathcal{G}_{1} \cup \mathcal{G}_{2}$ is the subgraph with vertex set $\mathcal{V}\left(\mathcal{G}_{1}\right) \cup \mathcal{V}\left(\mathcal{G}_{2}\right)$ and edge set $\mathcal{E}\left(\mathcal{G}_{1}\right) \cup \mathcal{E}\left(\mathcal{G}_{2}\right)$.

A path graph of $N$ vertices, denoted by $P_{N}$, is a graph whose vertices can be listed in the order $v_{1}$, $v_{2}, \ldots, v_{N}$ such that the edges are $\left(v_{i}, v_{i+1}\right)$ where $i=1,2, \ldots, N-1$. A path is a particularly simple example of a tree, since no vertex has degree 3 or more.

The Laplacian matrix $L(\mathcal{G}) \in \mathbb{R}^{N \times N}$ of a network of agents is defined as $L(\mathcal{G})=E(\mathcal{G}) E(\mathcal{G})^{\top}$, where $E(\mathcal{G})$ is the incidence matrix. The Laplacian matrix $L(\mathcal{G})$ is positive semidefinite, and if $\mathcal{G}$ is connected and undirected, then $0=\lambda_{1}(\mathcal{G})<\lambda_{2}(\mathcal{G}) \leq$ $\cdots \leq \lambda_{N}(\mathcal{G})$, where $\left\{\lambda_{i}(\mathcal{G})\right\}$ are the eigenvalues of $L(\mathcal{G})$.

The edge Laplacian is an edge variant of the graph Laplacian defined as $L_{e}(\mathcal{G})=E(\mathcal{G})^{\top} E(\mathcal{G})$, and has the following algebraic properties [16]: 1) The non-zero eigenvalues of $L_{e}$ are equal to the nonzero eigenvalues of $L ; 2$ ) the rank of $L_{e}$ depends only on the number of connected components; 3 ) the null space of $L_{e}, \mathcal{N}\left(L_{e}\right)$, depends on the number of cycles in the graph and it holds that $\mathcal{N}\left(L_{e}\right)=\mathcal{N}(E)$. Furthermore, $\mathcal{N}(E)$ is spanned by all the linearly independent signed path vectors corresponding to the cycles of $E$; 4$)$ if $\mathcal{G}$ is a spanning tree, then $L_{e}$ has no zero eigenvalues and, hence, $\mathcal{N}\left(L_{e}\right)=\emptyset$.

Finally, we denote by $|\cdot|$ the cardinality of a set (group of agents).

The next lemma follows from the results in [16] and will be useful in the reminder of this paper.

Lemma 1. Suppose $L_{e} \in \mathbb{R}^{N_{e} \times N_{e}}$ is the edge Laplacian of an undirected connected graph $\mathcal{G}$. Then, for allt $\geq 0$ and all vectors $z \in \mathbb{R}^{N_{e}}$ with $z=E^{\top} x$ and $x \in \mathbb{R}^{N}$, it holds that

$$
\left\|e^{-L_{e} t} z\right\| \leq e^{-\lambda_{2}(\mathcal{G}) t}\|z\| \text {. }
$$

Proof. Let us assume that the graph $\mathcal{G}$ has $n_{c}$ independent cycles. Then, the multiplicity of the zero eigenvalues of $L_{e}$ is $n_{c}$. Since $L_{e}$ is symmetric, the eigenvectors of $L_{e}$ can always be chosen such that they form and orthonormal basis $T$ and it holds that $L_{e}=T \cdot \operatorname{diag}\left(0, \ldots, 0, \lambda_{2}, \ldots, \lambda_{N}\right) \cdot T^{\top}$, where the first $n_{c}$ vectors in $T$ correspond to the $n_{c}$ zero eigenvalues, and the rest corresponds to the eigenvalues $\lambda_{2}, \ldots, \lambda_{N}$ of $L$. Then

$$
e^{-L_{e} t}=T \operatorname{diag}\left(1, \ldots, 1, e^{-\lambda_{2} t}, \ldots, e^{-\lambda_{N} t}\right) T^{\top} .
$$

For $z=E^{\top} x \in \mathbb{R}^{N_{e}}$, it holds

$$
\begin{aligned}
e^{-L_{e} t} z & =T \operatorname{diag}(1, \ldots, 1,0, \ldots, 0) T^{\top} z \\
& +T \operatorname{diag}\left(0, \ldots, 0, e^{-\lambda_{2} t}, \ldots, e^{-\lambda_{N} t}\right) T^{\top} z
\end{aligned}
$$

The first term is 0 using the third property described above and, hence

$\left\|e^{-L_{e} t} z\right\| \leq\left\|T \operatorname{diag}\left(0, \ldots, 0, e^{-\lambda_{2} t}, \ldots, e^{-\lambda_{N} t}\right) T^{\top} z\right\|$.

Note that $\|A \cdot B\| \leq\|A\|\|B\|$ for any two matrices and that $\|T\|=\left\|T^{\top}\right\|=1$ for an orthonormal basis, then it follows

$$
\begin{aligned}
\left\|e^{-L_{e} t} z\right\| & \leq\left\|\operatorname{diag}\left(0, \ldots, 0, e^{-\lambda_{2} t}, \ldots, e^{-\lambda_{N} t}\right)\right\|\|z\| \\
& =e^{-\lambda_{2} t}\|z\|,
\end{aligned}
$$

which completes the proof.

\section{PROBLEM STATEMENT}

\subsection{Control objective}

The control objective the system has to achieve is threefold:

- The group of agents, commanded by a subset of them called leaders, needs to reach a formation with relative position offset denoted by $d_{i j}=d_{i}-d_{j}$ around a point of the state space denoted by $c$. For each agent, we define a region of the state space $\mathcal{B}_{i}$ defined as

$$
\mathcal{B}_{i}=\left\{y \in \mathbb{R}^{n}:\left\|y-c_{i}\right\| \leq r\right\},
$$

and denoted as $\mathcal{B}_{i}=\left(c_{i}, r\right)$, where $c_{i}=c+d_{i}$.

- The group has to achieve such a control objective before some deadline denoted by $T$.

- The motion law is such that the connectivity of the group is maintained.

More details will be given in the following sections. 


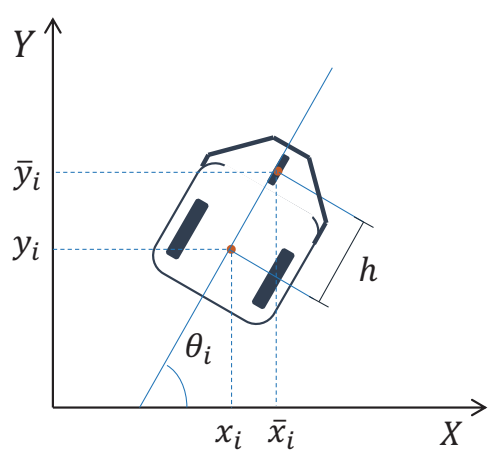

Figure 1: Scheme for a non-holonomic mobile robot.

\subsection{Agent dynamics and control}

Let us assume that the $N$ agents obey the singleintegrator dynamics:

$$
\dot{x}_{i}(t)=u_{i}(t), \quad i=1, \ldots, N,
$$

where $x_{i}(t), u_{i}(t) \in \mathbb{R}^{n}$ are the state and the control inputs of agent $i$, respectively. The state of the overall system is defined as $x=\left(x_{1}^{\top}, \ldots, x_{N}^{\top}\right)^{\top}$ and the control law $u=\left(u_{1}, \ldots, u_{N}^{\top}\right)^{\top}$.

For each agent of the form (2), let us consider a control law of the form

$$
u_{i}(t)=\kappa_{i}\left(x_{i}(t), \bigcup_{j \in \mathcal{N}_{i}(t)} x_{j}(t), \pi_{i}\right), \quad i=1, \ldots, N
$$

where each $\kappa_{i}(\cdot)$ is locally Lipschitz and $\pi_{i}$ represents a set of additional parameters. For instance, the leaders will include in $\pi_{i}$ information about the objective regions (1). The control law (3) requires the availability of the state of other agents, called neighbors and denoted by $\mathcal{N}_{i}$ for the agent $i$. Depending on the setup, this can be obtained from sensors (proximity sensors, for instance) or via communication. It is assume that an agent can only reach those others that stay inside a ball of radius $R$, which is the range of communication.

However, single integrators do not describe properly the dynamics of most of commercial mobile robots, since these cannot move in any direction instantaneously. In robotics, holonomicity refers to the relationship between the controllable and total degrees of freedom of a given robot. If the controllable degrees of freedom are less than the total degrees of freedom the vehicle is nonholonomic.

Let us consider a set of robots that are able to move in $\mathbb{R}^{2}$, i.e., its state can be described by its position in the plane and orientation $\left(x_{i}, y_{i}, \theta_{i}\right)$.
To avoid the non-holonomic constraint, let us define the dynamics in terms of the front wheels coordinates $\left(\bar{x}_{i}, \bar{y}_{i}\right)$ (see Figure 1$)$ :

$$
\left(\begin{array}{c}
\dot{\bar{x}}_{i} \\
\dot{\bar{y}}_{i} \\
\dot{\theta}_{i}
\end{array}\right)=\left(\begin{array}{cc}
\cos \theta_{i} & -h \sin \theta_{i} \\
\sin \theta_{i} & h \cos \theta_{i} \\
0 & 1
\end{array}\right)\left(\begin{array}{c}
v_{i} \\
\omega_{i}
\end{array}\right)
$$

where $\bar{x}_{i}=x_{i}+h \cos \theta_{i}, \bar{y}_{i}=y_{i}+h \sin \theta_{i}$, and $v_{i}$ and $\omega_{i}$ are the linear and angular velocities, respectively, and are considered the control iputs, $u_{i}=\left(v_{i}, \omega_{i}\right)^{\top}$.

According to [6], the following feedback linearization can be used to transform the dynamics (4) into two decoupled single integrators:

$$
\left(\begin{array}{c}
v_{i} \\
\omega_{i}
\end{array}\right)=\left(\begin{array}{cc}
\cos \theta_{i} & -h \sin \theta_{i} \\
\sin \theta_{i} & h \cos \theta_{i}
\end{array}\right)^{-1}\left(\begin{array}{c}
\bar{v}_{i} \\
\bar{\omega}_{i}
\end{array}\right) .
$$

With the new control inputs $\bar{v}_{i}, \bar{\omega}_{i}$, the linearized, decoupled model of robot $i$ is given by

$$
\left(\begin{array}{c}
\dot{\bar{x}}_{i} \\
\dot{\bar{y}}_{i}
\end{array}\right)=\left(\begin{array}{c}
\bar{v}_{i} \\
\bar{\omega}_{i}
\end{array}\right)
$$

and $\dot{\theta}_{i}=\omega_{i}$

Then, we can consider the single integrator model given by (2), and design the control law $u_{i}=$ $\left(\bar{v}_{i}, \bar{\omega}_{i}\right)$ to fulfill the control objectives and, then, implement it according to the transformation (5).

\section{MAIN CONTRIBUTION}

Next we present the control law that allow the achievement of the control objectives. Two type of agents are distinguished: the leaders, which know the final destination, and the rest of the agents called followers. We denote the leaders as $\mathcal{L}$. For simplicity, we assume that there is a single leader. Hence, we propose a continuous controller which may include one or two terms, depending on the role of the agent:

$u_{i}=\left\{\begin{array}{lr}\sum_{j \in \mathcal{N}_{i}} k\left(x_{j}-x_{i}-d_{j i}\right)-a\left(x_{i}-c\right) & \text { if } i \in \mathcal{L} \\ \sum_{j \in \mathcal{N}_{i}} k\left(x_{j}-x_{i}-d_{i j}\right) & \text { otherwise }\end{array}\right.$,

where $d_{j i}=d_{j}-d_{i} \in \mathbb{R}^{n}$ are predefined relative position offsets, and the gains $k$ and $a$ are to be designed such that the time constraint is fulfilled while the connectivity of the group is maintained.

The next two propositions give a guideline for the design of the feedback gains $k$ and $a$. If the dynamics are rewritten in terms of the group state $x$ and control vector $u$, then it follows

$\dot{x}=-k\left(L(\mathcal{G}) \otimes I_{n}\right)(x-d)-a\left(D \otimes I_{n}\right)\left(x-\mathbf{1}_{N} \otimes c\right)$, 
where $d=\left(d_{1}^{\top} \ldots d_{N}^{\top}\right)^{\top}, L(\mathcal{G})$ is the Laplacian matrix of the graph $\mathcal{G}$ that describes the topology, and $D$ is a diagonal matrix where its diagonal elements are defined as follows:

$$
D_{i i}= \begin{cases}1 & \text { if } i \in \mathcal{L} \\ 0 & \text { otherwise }\end{cases}
$$

Moreover, the incidence matrix allows rewriting the variables of the vertex $\mathcal{V}$ in terms of the edges $\mathcal{E}$. Actually, we can define a vector $z$ for the state of the edges in $\mathcal{E}$ such that $z=\left(E^{\top} \otimes I_{n}\right) x$, and its dynamics are given by

$$
\begin{aligned}
\dot{z}= & -k\left(L_{e}(\mathcal{G}) \otimes I_{n}\right)\left(z-d_{e}\right) \\
& -a\left(E^{\top}(\mathcal{G}) D \otimes I_{n}\right)\left(x-\mathbf{1}_{N} \otimes c\right),
\end{aligned}
$$

where $d_{e}=E^{\top} d$. Let us denote $\bar{z}=z-d_{e}$, then (10) is transformed into

$$
\begin{aligned}
\dot{\bar{z}}= & -k\left(L_{e}(\mathcal{G}) \otimes I_{n}\right) \bar{z} \\
& -a\left(E^{\top}(\mathcal{G}) D \otimes I_{n}\right)\left(x-\mathbf{1}_{N} \otimes c\right),
\end{aligned}
$$

We further make the following assumption.

Assumption 1. The distance to the objectives $c_{i}$ is upper bounded by $c_{\max }$, i.e., $\left\|x_{i}-c_{i}\right\| \leq c_{\max }$ $\forall i \in \mathcal{V}$. Furthermore, the initial conditions are such that $\|\bar{z}(0)\| \leq R$, where $R$ is the communication range.

Proposition 1. If the graph $\mathcal{G}$ is connected at $t=$ 0 and Assumption 1 holds, the graph $\mathcal{G}$ remains connected and no links are removed if the feedback gains $k$, a in (7) satisfy the following relationship:

$$
a \leq \frac{R \lambda_{2}(\mathcal{G}) k}{\sqrt{N} c_{\max }} .
$$

Proof. Let us assume that no edges are removed or added in the group of agents. Thus, according to (11), the evolution of the system is

$$
\begin{aligned}
& \bar{z}(t)=e^{-k\left(L_{e} \otimes I_{n}\right) t} \bar{z}(0) \\
& -\int_{0}^{t} e^{-k\left(L_{e} \otimes I_{n}\right)(t-s)} a\left(E^{\top} D \otimes I_{n}\right)\left(x-\mathbf{1}_{N} \otimes c\right) d s .
\end{aligned}
$$

Taking norms and using the result of Lemma 1, it follows that

$$
\begin{aligned}
\|\bar{z}(t)\| & \leq e^{-k \lambda_{2} t}\|\bar{z}(0)\| \\
& +a \sqrt{N} \int_{0}^{t} e^{-k \lambda_{2}(t-s)} \sup _{i \in \mathcal{V}}\left\|x_{i}-c\right\| d s .
\end{aligned}
$$

From Assumption 1, $\sup _{i \in \mathcal{V}}\left\|x_{i}-c\right\| \leq c_{\max }$ and $\|\bar{z}(0)\| \leq R$ and, hence

$$
\|\bar{z}(t)\| \leq e^{-k \lambda_{2} t} R+\frac{a \sqrt{N} c_{\max }}{k \lambda_{2}}\left(1-e^{-k \lambda_{2} t}\right) .
$$

The connectivity is maintained if $\left\|\bar{z}_{i}(t)\right\| \leq R$ for all the edges $i$ in $\mathcal{E}$. Since $\left\|\bar{z}_{i}(t)\right\| \leq\|\bar{z}(t)\|$, the feedback gain $a$ given by (12) guarantees the connectivity and that no edges are removed.
Remark 1. In the proof of Proposition 1, it is assumed that no edges are added to the graph $\mathcal{G}$. This can be relaxed without altering the results, since adding new edges in the graph can only increase the value of $\lambda_{2}(\mathcal{G})$ [16] and, hence, all the upper bounds used in the proof still hold if the value of $\lambda_{2}(\mathcal{G})$ at the beginning of the task is considered.

Before proceeding with the results that guarantee that the systems completes its objective on time, the following lemma studies the spectral properties of the matrix $M=k L+a D \in \mathbb{R}^{N \times N}$, $k, a \in \mathbb{R}_{>0}$, where $L$ and $D$ are the Laplacian matrix and $D$ isdefined in (9).

Lemma 2. The eigenvalues of the matrix $M$ defined above are lower bounded by

$$
\left(\frac{N-1}{N(N-1)+a / k}\right)^{N-1} a .
$$

Proof. The proof is provided in the appendix.

Since it is assumed there is a single leader in the group, i.e., the number of elements of the set $\mathcal{L}$ is 1. This simplifies the analysis, but the results can be extended to a more general setting.

Proposition 2. If the feedback gains $k$, a in (7) are chosen such that

$$
\left(\frac{N-1}{N(N-1)+a / k}\right)^{N-1} \geq \frac{1}{T} \log \frac{\sqrt{N} c_{\max }}{r},
$$

then the team of agents reaches the formation defined by the set of region $\mathcal{B}_{i}$ given in (1) before $T$.

Proof. Let us consider the group dynamics (8), which can be rewritten as follows

$\dot{x}=-k\left(L(\mathcal{G}) \otimes I_{n}\right)(x-d)-a\left(D \otimes I_{n}\right)\left(x-d-\mathbf{1}_{N} \otimes c_{\mathcal{L}}\right)$, where $c_{\mathcal{L}}=c-d_{\mathcal{L}}$, being $d_{\mathcal{L}}$ the offset position of the leader $\mathcal{L}$. Since $\left(L(\mathcal{G}) \otimes I_{n}\right)\left(\mathbf{1}_{N} \otimes c_{\mathcal{L}}\right)=0\left(\mathbf{1}_{N}\right.$ is an eigenvector of $L(\mathcal{G})$ ), then

$$
\dot{x}(t)=-(k L(\mathcal{G})+a D) \otimes I_{n}\left(x(t)-d-\mathbf{1}_{N} \otimes c_{\mathcal{L}}\right) .
$$

If we define $\delta(t)=x(t)-d-\mathbf{1}_{N} \otimes c_{\mathcal{L}}$, it follows that

$$
\dot{\delta}(t)=-(k L(\mathcal{G})+a D) \otimes I_{n} \delta(t) .
$$

If we denote as $x_{0}\left(\delta_{0}=x_{0}-d-\mathbf{1}_{N} \otimes c_{\mathcal{L}}\right)$ and $t_{0}=0$ the initial conditions and the starting time, respectively, then it holds that

$$
\delta(t)=e^{-\left((k L(\mathcal{G})+a D) \otimes I_{n}\right) t} \delta_{0} .
$$

Let us denote $M=(k L(\mathcal{G})+a D) \otimes I_{n}$ and we next apply the results of Lemma 2 to this matrix. 
Note that the eigenvalues of $e^{-(k L(\mathcal{G})+a D) \otimes I_{n}}$ are all negative (the eigenvalues of $M$ are all positive), and this guarantees that $\delta(t)$ in (17) converges to zero $(\delta \rightarrow 0$ when $t \rightarrow \infty)$. Moreover, because $M$ has an orthonormal basis of eigenvectors, it holds that $\|\delta(t)\|=\left\|e^{-\left((k L(\mathcal{G})+a D) \otimes I_{n}\right) t} \delta_{0}\right\| \leq$ $e^{-\lambda_{1}(M) t}\left\|\delta_{0}\right\| \leq e^{-\underline{\lambda_{1}}(M) t}\left\|\delta_{0}\right\|$, where

$$
\underline{\lambda_{1}}(M)=\left(\frac{N-1}{N(N-1)+a / k}\right)^{N-1} a,
$$

according to Lemma 2. Thus, in particular, for $t=T$ :

$$
\|\delta(T)\| \leq e^{-\underline{\lambda_{1}}(M) T}\left\|\delta_{0}\right\| .
$$

If $k, a$ fulfill (14), then all agents reach the boundary of $\mathcal{B}_{i}$ before $T$.

Remark 2. Propositions 1 and 2 provide constraints for the choice of $a$ and $k$ to guarantee that the graph remains connected and the objective region is achieved on time. For instance, (12) imposes $a / k$ to be upper bounded by $\alpha=\frac{R \lambda_{2}(\mathcal{G})}{\sqrt{N} c_{\max }}$. Then, taking the equality and introducing it in (14) yields

$$
\begin{aligned}
& a=\left(\frac{N(N-1)+\alpha}{N-1}\right)^{N-1} \frac{1}{T} \log \frac{\sqrt{N} c_{\max }}{r} \\
& k=\frac{1}{\alpha} a
\end{aligned}
$$

Moreover, to obtain feasible values of $k$ and $a$, it is required that $r<\sqrt{N} c_{\max }$, which can be guaranteed by an appropriate choice of $c_{\max }$. For instance, $c_{\max }$ is an upper bound for the distance to the objective regions and can be increased to satisfy the constraint.

Remark 3. The value of $k$ given by (20) requires the knowledge of the eigenvalue $\lambda_{2}(L(\mathcal{G}))$. This can be estimated in a distributed fashion as proposed in [1]. Alternatively, one can consider an upper bound such as $\lambda_{2}(L(\mathcal{G})) \leq \min _{v \in \mathcal{V}} \operatorname{deg}(v)$, i.e., the minimum degree of the graph.

\section{SIMULATION EXAMPLE}

Let us consider a system of four vehicles whose dynamics are modeled by (4) with $h=0.16$, and whose communication graph is characterized by the following Laplacian matrix

$$
L=\left(\begin{array}{cccc}
2 & -1 & 0 & -1 \\
-1 & 2 & -1 & 0 \\
0 & -1 & 2 & -1 \\
-1 & 0 & -1 & 2
\end{array}\right)
$$

and thus, $\lambda_{2}(L)=2$. The desired formation is described in Figure 2, and the point $c$ in the plane around the formation should be situated is $c=$ $(-2,5)$. The leader of the formation is the agent 1. The rest of the parameters of the setting are

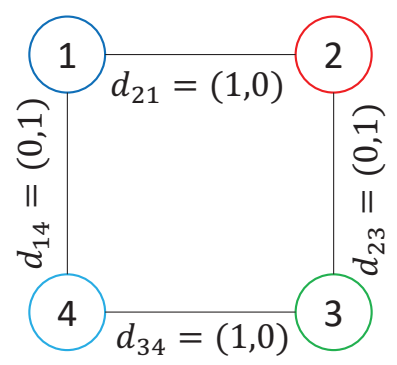

Figure 2: Relative distance of the desired formation and communication graph.

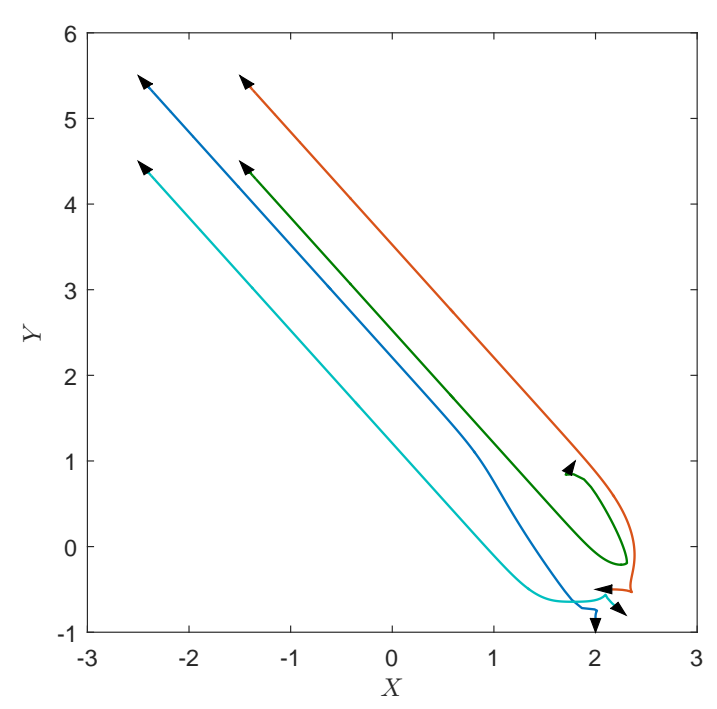

Figure 3: Movement of the robots in the plane.

$r=0.05, R=3$, and $c_{\max }=10$. The deadline for reaching the formation described by the set of regions $\mathcal{B}_{i}$ as in (1) is set to $T=10 \mathrm{~s}$. The initial conditions are $x_{0}^{\top}=(2-12-0.52 .3-0.81 .8-1)$, and the orientations $\theta_{i}$ of the vehicles $1,2,3$, and 4 are initially set to $-\pi / 2, \pi,-\pi / 4$, and $\pi / 3$, respectively.

If feedback gains $k$ and $a$ are computed according to (20) and (21), respectively, the result is $k=91.76$ and $a=27.53$. The trajectory of the robots in the plane is depicted in Figure 3. It can be noticed that the robots go to the final destination point and the desired formation is achieved. Additionally, note how the non-holonomic dynamics affects the movement of the vehicles, since the robots first have to change their orientation and then moves towards their objective. In Figure 4, the disagreement respect the desired formation $\delta$ is depicted. Note how the disagreement converges to zero very fast and, hence, the time constraint is satisfied by far. 


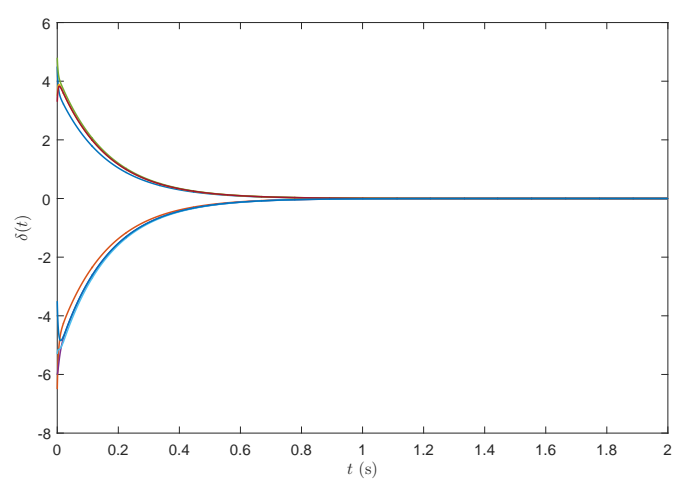

Figure 4: Time evolution of the disagreement respect to the desired formation.

\section{DISCUSSION}

In this paper, by analyzing the rate of convergence of the system, we derive distributed control laws that guarantee that the formation of a team of robots is achieved on time while the connectivity of the group is maintained. The simulation example illustrates the analytical results and shows that the rate of convergence if much faster than the imposed by the time constraint. This is due to the possible conservatism in the bounds derived for certain parameters of the overall system dynamics, that depend on the number of agents. Future research will include the study of other methods that scale better with the dimension of the group and the extension to other types of robots that have more degrees of freedom such as quadcopters.

\section{Acknowledgments}

Work funded by the Spanish Ministry of Education, Culture, and Sport (MECD) under grant JC2015-0041 and the Spanish Ministry of Economy and Competitiveness under contracts DPI2014-55932-C2-2-R and DPI2017-84259-C2-2$\mathrm{R}$.

\section{APPENDIX}

Proof of Lemma 2: The Laplacian matrix $L$ has eigenvalues $0=\lambda_{1}(L)<\lambda_{2}(L) \leq \ldots \leq \lambda_{N}(L)$. Furthermore, $D$ has one eigenvalue equal to 1 , and the rest $N-1$ of them are zero. Note that $M$ is positive definite by construction, and thus all its eigenvalues are real and positive. Then, the results of Lemma 1 in [7] apply, i.e., for any matrix $A \in \mathbb{C}^{n \times n}$ with real and positive eigenvalues it holds that

$$
\lambda_{1}(A) \geq\left(\frac{n-1}{\operatorname{tr}(A)}\right)^{n-1} \operatorname{det}(A)
$$

where $\lambda_{1}(A)$ is the smallest eigenvalue of $A$.
The trace of the matrix $M$ is upper bounded by

$$
\operatorname{tr}(M) \leq k N(N-1)+a>0,
$$

since the diagonal elements of $L$ are the degree of each vertex which is upper bounded by $N-1$.

We next find a lower bound for the determinant of $M$. The determinant of $M$ is the product of its eigenvalues and, thus, is positive. From Weyl's theorem [5], it holds that $0<\lambda_{1}(M) \leq k \lambda_{2}(L)$, $k \lambda_{N}(L) \leq \lambda_{N}(M)$, and $k \lambda_{i}(L) \leq \lambda_{i}(M) \leq$ $k \lambda_{i+1}(L) \forall i=2, \ldots, N-1$ (the eigenvalues interlace). Furthermore, according to Theorem 4.1 in [16], the eigenvalues of a connected graph Laplacian $L$ are lower bounded by those of the contained spanning trees. Hence, we next analyze the case of $M$ when the graph is a tree and, for simplicity, a path graph, and we show that adding an edge in the graph can only increase the determinant. The extension to a general tree is trivial.

Let us consider the path graph $P_{2}$ and let us assume, without loss of generality, that $D=$ $\operatorname{diag}(1,0)$. Then

$$
\operatorname{det}(M)=\left|\begin{array}{cc}
k+a & -k \\
-k & k
\end{array}\right|=k a=k^{2-1} a>0 .
$$

Similary, for $P_{3}$ and $D=\operatorname{diag}(1,0,0)$ it holds

$$
\operatorname{det}(M)=\left|\begin{array}{ccc}
k+a & -k & 0 \\
-k & 2 k & -k \\
0 & -k & k
\end{array}\right|=k^{2} a=k^{3-1} a>0 .
$$

Assume that this holds for $N-1$, that is, for $L=$ $P_{N-1}, \operatorname{det}(M)=k^{N-2} a$. Then, for $L\left(P_{N}\right)$ and $D=\operatorname{diag}(1,0, \ldots, 0) \in \mathbb{R}^{N \times N}$ :

$\operatorname{det}(M)=\left|\begin{array}{cccc}k+a & -k & \ldots & 0 \\ -k & 2 k & \ldots & 0 \\ \vdots & \vdots & \ddots & \vdots \\ 0 & 0 & \ldots & k\end{array}\right|=\left|\begin{array}{cccc}a & -k & \ldots & 0 \\ 0 & 2 k & \ldots & 0 \\ \vdots & \vdots & \ddots & \vdots \\ 0 & 0 & \ldots & k\end{array}\right|$

where we have used basic properties of the determinants in the last equality. Applying the Laplace expansion by minors along the first row [5]:

$\operatorname{det}(M)=a \cdot\left|\begin{array}{cccc}2 k & -k & \ldots & 0 \\ -k & 2 k & \ldots & 0 \\ \vdots & \vdots & \ddots & \vdots \\ 0 & 0 & \ldots & k\end{array}\right|+k \cdot\left|\begin{array}{cccc}0 & -k & \ldots & 0 \\ 0 & 2 k & \ldots & 0 \\ \vdots & \vdots & \ddots & \vdots \\ 0 & 0 & \ldots & k\end{array}\right|$.

The second determinant is zero, since the first column has all its elements equal to zero. For the first determinant, the element in the first row and the first column can be decomposed as $2 k=k+k$ and then $\operatorname{det}(M)=a \operatorname{det}\left(k L\left(P_{N-1}\right)+k \cdot D_{N-1}\right)$. Then, using the results for the case $N-1$ with $a=k$ it follows that

$$
\operatorname{det}(M)=k^{N-1} a .
$$


Assume that an edge is added to $P_{N}$ and denote this graph by $P_{N}^{+e}$. Then the incidence matrix of $P_{N}^{+e}$ is $E\left(P_{N}^{+e}\right)=\left[E\left(P_{N}\right) e\right]$ and the Laplacian matrix $L\left(P_{N}^{+e}\right)=L\left(P_{N}\right)+e e^{\top}$, where $e^{\top}=$ $\left(e_{1} \ldots e_{N}\right)$ denotes a column corresponding to the added edge and $e_{i}=1$ if $i$ is the initial node of the edge, $e_{i}=-1$ if $i$ is the terminal node of the edge, otherwise $e_{i}=0$. Thus, in this case, the matrix $M$ can be written as $M=k\left(L\left(P_{N}\right)+e e^{\top}\right)+a D_{N}$. Using the fact that $\operatorname{det}(A+B) \geq \operatorname{det}(A)+\operatorname{det}(B)$, it follows that adding new edges in the graph can only increase the determinant and (24) is a lower bound: $\left(\operatorname{det}\left(k\left(L\left(P_{N}\right)+e e^{\top}\right)+a D_{N}\right) \geq\right.$ $\left.\operatorname{det}\left(k\left(L\left(P_{N}\right)+a D_{N}\right)\right)+\operatorname{det}\left(e e^{\top}\right)=k^{N-1} a\right)$. Combining this with (23) and (22), it yields

$$
\left(\frac{N-1}{N(N-1) k+a}\right)^{N-1} k^{N-1} a \leq \lambda_{1}(M) \leq \cdots \leq \lambda_{N}(M),
$$

which completes the proof.

\section{References}

[1] R. Aragues, G. Shi, D.V. Dimarogonas, C. Sagüés, K.H. Johansson, and Y. Mezouar. Distributed algebraic connectivity estimation for undirected graphs with upper and lower bounds. Automatica, 50(12):3253-3259, 2014.

[2] J. Cortes. Finite-time convergent gradient flows with applications to network consensus. Automatica, 42(11):1993 - 2000, 2006.

[3] J. Cortes, S. Martinez, and F. Bullo. Spatially-distributed coverage optimization and control with limited-range interactions. ESAIM: Control, Optimisation and Calculus of Variations, 11(4):691â"719, 2005.

[4] T. Gustavi, D.V. Dimarogonas, M. Egerstedt, and X. Hu. Sufficient conditions for connectivity maintenance and rendezvous in leaderfollower networks. Automatica, 46(1):133 139, 2010.

[5] R.A. Horn and C.R. Johnson. Matrix analysis. Cambridge university press, 2012.

[6] J. R. T. Lawton, R. W. Beard, and B. J. Young. A decentralized approach to formation maneuvers. IEEE Transactions on Robotics and Automation, 19(6):933-941, 2003.

[7] J.K. Merikoski and A. Virtanen. Bounds for eigenvalues using the trace and determinant. Linear Algebra and its Applications, 264(Supplement C):101 - 108, 1997.
[8] R. Olfati-Saber, J. A. Fax, and R. M. Murray. Consensus and cooperation in networked multi-agent systems. Proceedings of the IEEE, 95(1):215-233, 2007.

[9] L. C. A. Pimenta, V. Kumar, R. C. Mesquita, and G. A. S. Pereira. Sensing and coverage for a network of heterogeneous robots. In 2008 47th IEEE Conference on Decision and Control, pages 3947-3952, 2008.

[10] M. Schuresko and J. Cortes. Safe graph rearrangements for distributed connectivity of robotic networks. In 2007 46th IEEE Conference on Decision and Control, pages 46024607, 2007.

[11] M. Schwager, D. Rus, and J.J. Slotine. Decentralized, adaptive coverage control for networked robots. The International Journal of Robotics Research, 28(3):357-375, 2009.

[12] H. Su, X. Wang, and G. Chen. Rendezvous of multiple mobile agents with preserved network connectivity. Systems \& Control Letters, 59(5):313 - 322, 2010.

[13] F. Xiao, L. Wang, J. Chen, and Y. Gao. Finite-time formation control for multi-agent systems. Automatica, 45(11):2605 - 2611, 2009 .

[14] M. M. Zavlanos, A. Jadbabaie, and G. J. Pappas. Flocking while preserving network connectivity. In 2007 46th IEEE Conference on Decision and Control, pages 2919-2924, 2007.

[15] M. M. Zavlanos and G. J. Pappas. Distributed connectivity control of mobile networks. IEEE Transactions on Robotics, 24(6):1416-1428, 2008.

[16] D. Zelazo, A. Rahmani, and M. Mesbahi. Agreement via the edge laplacian. In Decision and Control (CDC), 2007 IEEE 46th Annual Conference on, pages 2309-2314. IEEE, 2007.

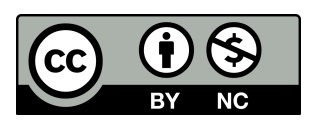
(c) 2018 by the authors. Submitted for possible open access publication under the terms and conditions of the Creative Commons Attribution CC-BY-NC 3.0 license (http://creativecommons.org/licenses/by-nc/3.0/). 